\title{
Do Medical Residents with Rural Upbringing Show Less Decline in Empathy During Training? A Report from a Rural Family Medicine Residency
}

\author{
William J. Crump, MD' ${ }^{1}$ Craig H. Ziegler, PhD², R. Steve Fricker, MPA ${ }^{1}$
}

\section{ABSTRACT}

Purpose: Our focus in this study was to determine if demographic variables, including rural upbringing, showed any association with a measure of empathy among family medicine residents at a rural site.

MetHods: We surveyed 40 residents annually using the Jefferson Scale of Empathy (JSE) from 2016 to 2020 and compared scores between residents with rural vs. urban hometowns. The response rate was 98/99 (99\%) of completed survey opportunities.

FINDINGS: There was no significant difference in JSE scores of rural vs. urban residents at baseline, and both groups showed a decline after the PG-1 year. However, the ruralraised residents showed a significantly different rebound after the PG-2 and PG-3 years, with the urban-raised residents showing a slight continuing decline $(p=.023$ and $\mathrm{p}=.033)$.

CONCLUSION: These preliminary findings among family medicine residents at a rural site suggest that residents with rural backgrounds might regain empathy better than residents with urban backgrounds during their training. Further study should validate our findings and address possible explanations, including the importance of cultural concordance with the patient population served. This and other hypotheses will be explored in further studies with focus groups and other contemporaneous measures.

\section{KEYWORDS}

Rural upbringing, medical resident training, empathy

\section{INTRODUCTION}

Most of the focus of rural medical education has concerned the inadequate supply of physicians in rural areas. Studies have shown that students who grow up in small towns are more likely to practice in similar-sized towns. ${ }^{1-4}$ Reports have also shown that rural students are more likely to have lower standardized test scores at admission but catch up rapidly during medical school. ${ }^{5-7}$ Students who train in rural locations are also more likely to choose rural locations for practice. . $^{4,7}$

Similarly, there is evidence that medical residents who have rural upbringing are more likely to return to similar-sized towns to practice. Like the student data, residents trained in a rural setting are likely to practice later in rural towns. ${ }^{9-11}$

Regardless of practice site, empathy is considered essential for all physicians. Measures of empathy using the Jefferson Scale of Empathy (JSE) have shown similar findings in Europe and Asia to those in the United States and some correlation with patient outcomes. The JSE is by far the most frequently used measure, with previous studies reporting gender and age in 10-year increments; however, there are no reports comparing rural vs. urban upbringing. ${ }^{12-13}$ 
We recently began to report a longitudinal study that collected six years of empathy measures from medical students based at our rural, regional campus using the JSE. Compared to students who trained in urban environments, we found that our students started medical school with similar JSE scores but showed significantly higher empathy scores after the second and third years of medical school compared to urban campuses, where students declined significantly. ${ }^{14}$

We recently reported a summary of all studies using the JSE among American residencies and compared the total JSE scores of our longitudinal residency study to these. ${ }^{15,16}$ Previous studies showed a decline in JSE score across the training period; however, these were cross-sectional, and all were urban sites. Our study was one of only two published designs that allowed us to do longitudinal analysis with individually-paired results, while others were only reports of means. In that previous study, we found by using paired analysis among our residents, about one-third showed little change across the training years, and $22 \%$ increased their JSE scores. ${ }^{15}$ We concluded that empathy measures vary among subsets of residents, with some drifting downward to cynicism, others recovering, and some increasing measures of empathy across training.

Driven by those findings, the purpose of this study was to determine if we could explain the difference in the subsets by gender, as previous studies among medical students had shown that women had higher total JSE scores. ${ }^{13}$ We also hypothesized that older residents with more life experience prior to beginning residency might have higher scores, and we wondered if ethnicity might be associated as well. Given our site's focus on rural training, we included rural upbringing as a demographic variable.

\section{METHODS}

\section{Setting and Participants}

Our program is based in a town of 20,000 in a rural area in the upper southeast, with six residents in each year, with no other residencies in town. It began in 1971 , becoming the 85th family medicine program in the United States and the first in the state. ${ }^{17}$ The mission is focused on providing rural family physicians for our region. At the last report, $49 \%$ of graduates remain in rural practice, and $41 \%$ were practicing within 100 miles of the program. The host hospital is part of a statewide system, with 312 licensed beds and 140 in operation. At last report, there were 5,189 annual discharges, 26,000 ED visits, 40,000 outpatient visits, and 640 births. The site is also host for our regional rural medical school campus, with the main campus in a metropolitan community 160 miles away. ${ }^{4}$

Beginning in 2016, 40 residents who were born in the U.S. and could have their hometown classified completed the empathy survey either just as the new academic year began or just as it ended, resulting in an annual survey for each resident. This allowed a measure of potential changes across training classes as training progressed. Each survey had the resident's name included for later matching to demographic variables, and residents placed the completed surveys into an envelope confidentially. Participants were assured that a research assistant unknown to them would then assign their ID number, and subsequently, no one would be able to connect their responses to their name. The host hospital IRB designated the project as exempt.

\section{Instrument and Procedures}

The JSE has 20 items, each ranked on a Likert scale from 1 to 7, with higher scores representing more measured empathy. It has reversed items that are accounted for in the total scoring. Subscales are not reported, and it has a normal distribution. ${ }^{13}$ The JSE has been used previously to assess changes during residency training in a cross-sectional fashion, as we did in this study. ${ }^{18-20}$

Each resident completed the JSE annually, including a baseline as their residency began. In a cross-

sectional analysis, each training year was considered individually, and there was no matching by individual resident in this study. The results reported by year of training included those in the graduating classes of 2016-2023, with the full complement of all four surveys reported within each cross-section for the classes of 2019-2020. We drew resident self-reported demographics and hometowns from their applications and summarized these using 
frequencies and percentages.

\section{VARIABLES}

Each JSE score was allocated to the postgraduate year just completed, with results from a range of 23-27 residents available for each training year. As independent variables, we included gender as male or female, race/ethnicity as White, Asian, or Hispanic (no African Americans were in residency program during the study years), age as less than 29 or 29 and over at the time of residency entry, and rural or urban upbringing, defining rural as a hometown population of $<30,000$ and a non-metro Rural-Urban Continuum Code (RUCC). ${ }^{21}$

\section{AnALYsIS}

Histograms for baseline, post-PGY 1, post-PGY 2, and post-PGY 3 scores were examined, along with the mean, median, standard deviation, skewness, and kurtosis. Histograms showed a normal distribution for all training years (including baseline) and no skewness or kurtosis, except for post-PGY 2, where there was a slightly platykurtic distribution. Demographics were tabulated using frequency and percentages.

A one-way analysis of variance (ANOVA) was used to test differences across training years using a Welch correction. Data for the analysis was not matched, as residents might have completed the JSE at some training years but not during other periods. Hence, a matching analysis would have led to the elimination of most observations.

JSE was compared by rural upbringing, gender, age dichotomized as $<29$ or $\geq 29$ years, and race at each training period using either Welch's t-tests or a one-way ANOVA with Welch's correction. We used the Fisher-Freeman-Halton chi-square Exact test to compare rural versus urban upbringing with the demographic variables.

We used a bar graph to display differences in JSE mean scores by training year. A similar bar graph was used to show the JSE total mean score by rural versus urban upbringing for each training year. We used IBM SPSS Statistics for Windows (version 26.0, 2019, IBM Corporation, Armonk, NY, 877-426-6006) and the website statpages.info20 to analyze the data. We created figures with the R package ggplot $2 .{ }^{22}$ Statistical significance was set by convention at $p$ $<0.05$.

\section{RESULTS}

One resident missed completing a post-PG-2 survey during the study period because of an extended leave. All others completed the survey per an annual administrative routine, making the final response rate 98 of 99 opportunities (99\%).

The demographics of all the residents included in this study, regardless of training year, are shown in table 1. National demographics for race and rurality

\begin{tabular}{|l|l|c|c|}
\hline Gender & Males [Freq (\%)] & 26 & $(65 \%)$ \\
& Females [Freq (\%)] & 14 & $(35 \%)$ \\
\hline \multirow{2}{*}{ Race } & & & \\
& White [Freq (\%)] & 21 & $(53 \%)$ \\
& Asian [Freq (\%)] & 15 & $(38 \%)$ \\
\hline & Hispanic [Freq (\%)] & 4 & $(10 \%)$ \\
\hline \multirow{2}{*}{ Age 29 or older } & & & \\
& Yes [Freq (\%)] & 22 & $(55 \%)$ \\
& No [Freq (\%)] & 18 & $(45 \%)$ \\
\hline Rural & & & \\
& Yes [Freq (\%)] & 11 & $(28 \%)$ \\
\hline & No [Freq (\%)] & 29 & $(73 \%)$ \\
\hline
\end{tabular}

TABLE 1. Demographics of Residents $(\mathrm{N}=40)$

aRural was defined as a hometown population of $<30,000$ and a non-metro Rural-Urban Continuum Code (RUCC). ${ }^{21}$ 


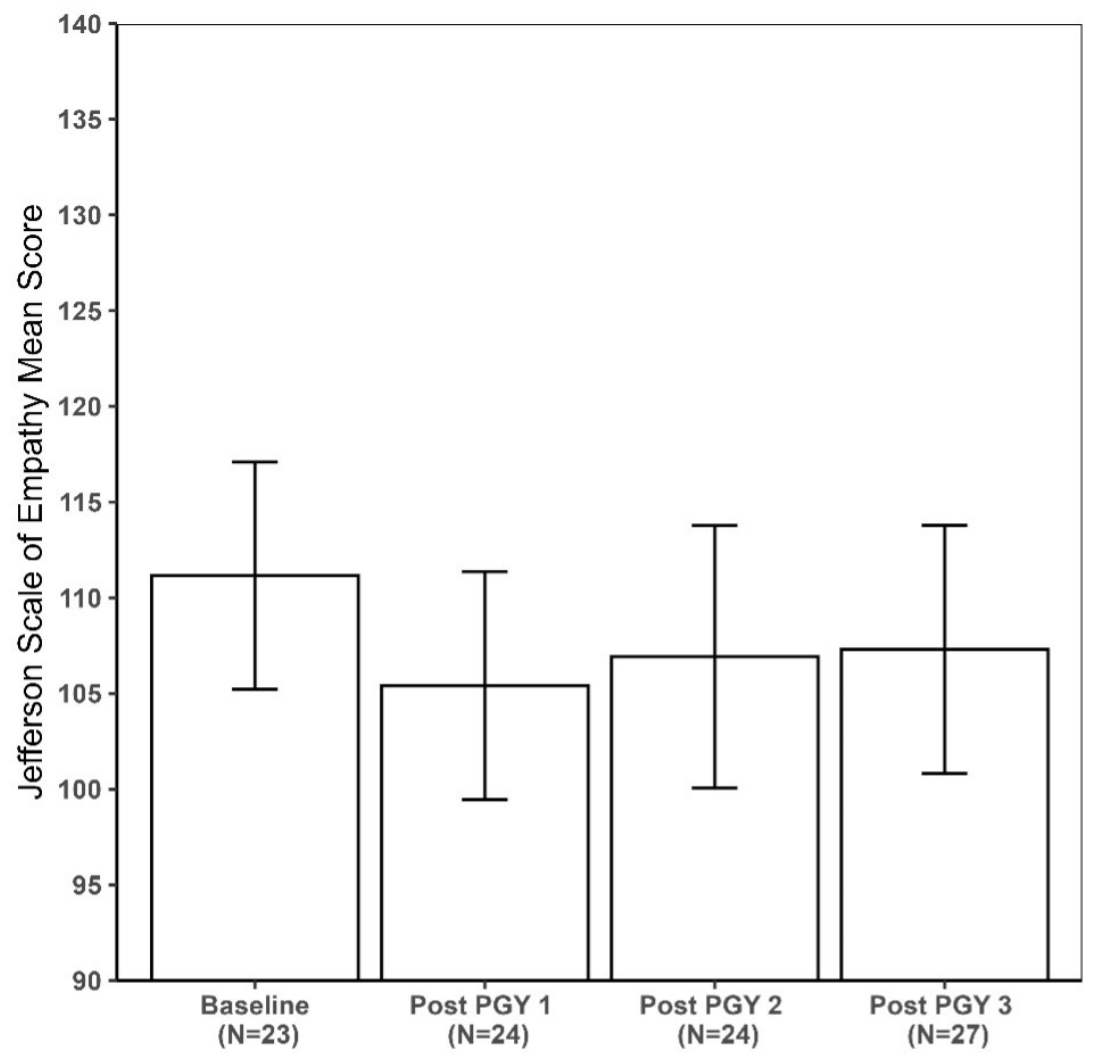

FIGURE 1. JSE score for all residents by year of training. Error bars reflect $95 \%$ confidence intervals, $F(3,52.06)=698, p=0.558$.

of family medicine residents are not available. However, our mean age of 30 is the same as for all family medicine residents reported nationally and about two years younger than available data on all rural residencies. ${ }^{23}$ The national proportion of men in family medicine residencies is $46 \%$, and ours of $65 \%$ is closer to the proportion reported for all rural residencies, which is $57 \%$.

When all training years were combined, there were no significant associations of total JSE score with traditional demographics (gender, race/ethnicity, age; data not shown). Figure 1 shows the mean JSE total score for residents by training year. There was a non-significant decrease from baseline at the beginning of residency to the end of the PG-1 year and then a slight recovery, again non-significant, over the next two years. For comparison to the JSE scores shown for our study population, we found only two reports from residencies that included JSE scores for each training year in enough detail to be useful. The most similar report from a community hospital setting in Flint, Michigan showed the following means and standard deviations: baseline: 110(14.6), post-PG-1: 117(9.8), post-PG-2: 108(14.1), post-PG-3:114(11.4). ${ }^{19}$

Figure 2 shows the mean JSE score by training year comparing those with rural vs. urban upbringing. There was no significant difference at baseline, and both groups showed a decline after the PG-1 year. However, the rural-raised residents showed a significantly different rebound after the PG-2 and PG-3 years, with the urban-raised residents showing a slight continuing decline. At baseline, although the difference in the JSE for rural-raised and urban residents was not significant, the difference of 6.97 (95\% Cl: $-8.08,22.02, \mathrm{p}=0.312$ ) might be important, and the result could be a function of a small sample. There were no significant associations of JSE score with traditional demographics (gender, age, and race/ethnicity) by training year. A comparison of rural vs. urban demographics showed the rural and urbanraised residents had no differences in traditional demographic variables (data not shown, $p$ values $>0.20)$, except for race. Ninety-one percent $(10 / 11)$ 


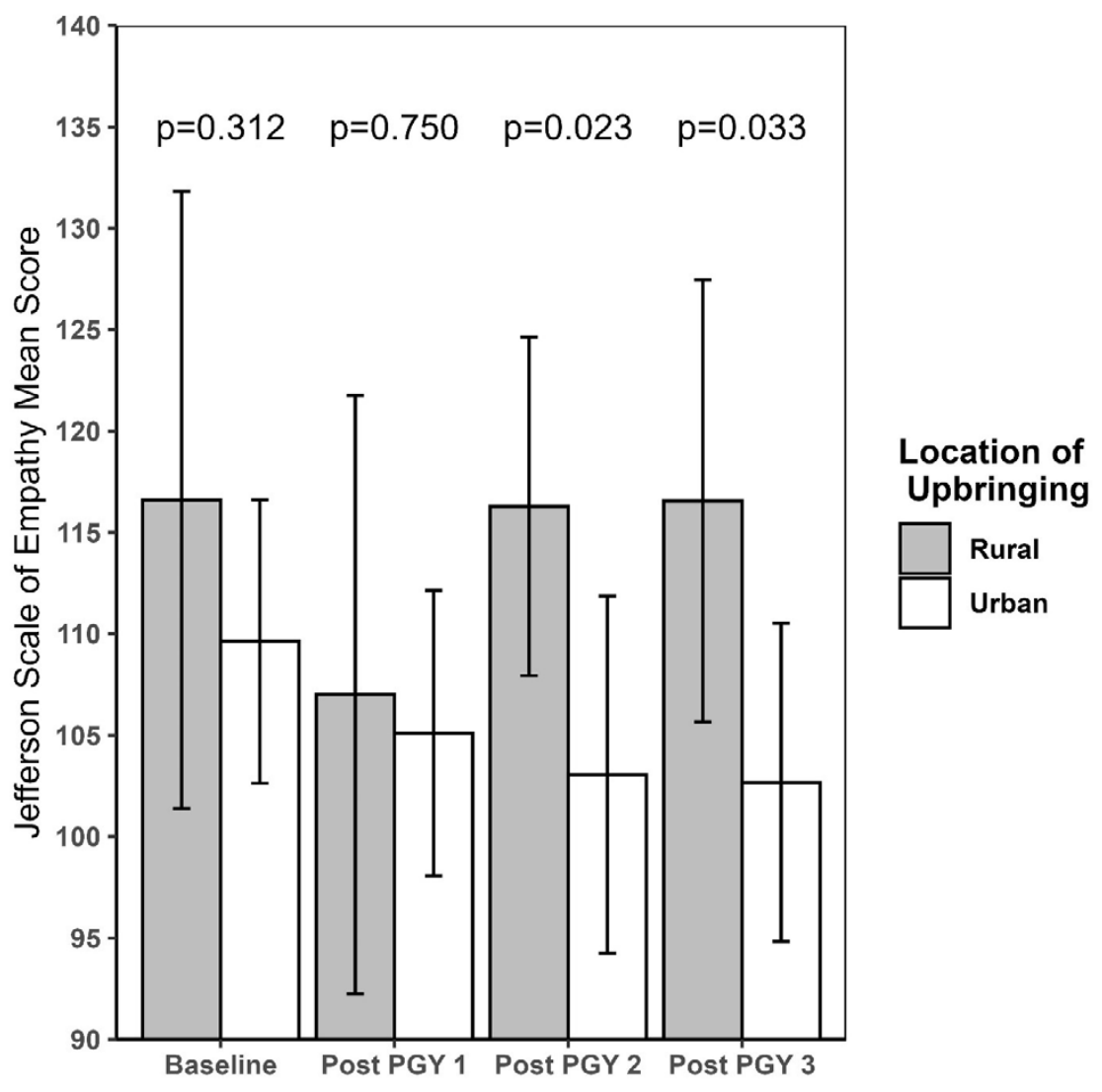

FIGURE 2. JSE score for all residents by rural or urban upbringing. Error bars reflect $95 \%$ confidence intervals.

of residents from rural areas were white, compared to $38 \%(11 / 29)$ of residents who had an urban upbringing, $p=0.011$. These numbers were deemed too small for further sub-analysis.

\section{DISCUSSION}

Despite many publications citing a decline in measured empathy across medical school and residency, the validity of these studies has recently been questioned. ${ }^{24}$ In our previous reports from this residency using longitudinal paired results across training, we found subsets that showed a decline, an increase, and no change in JSE scores. ${ }^{15,16}$ In the current study, we could not explain these subsets using traditional demographics but did find a difference in scores based on rural upbringing. Our findings must be considered exploratory, as ideally, a more extensive data set would be used to adjust for the effects of all demographics, including rural upbringing, on the JSE score. Ultimately, a data set large enough to do a true multivariate analysis would be ideal.

Looking at the literature on empathy among medical residents with rural upbringing, we found nothing published in English. We found one review that included 209 studies, including those in Spanish, Portuguese, French, and English, and none reported rural upbringing as a variable. ${ }^{25}$ Our previous report with student JSE was the first to include training at a rural campus as a variable. It showed that our students had higher JSE scores than those trained in urban environments, but we had too few urbanraised students to analyze using upbringing as a variable. ${ }^{14}$ We found no reports of student JSE scores that included rural upbringing as a variable.

Limitations AND StREnGthS

As with all previous reports on resident empathy, 
single-site studies are subject to selection bias and limited generalizability. Residents who seek and subsequently match to a rural, regional hospital in an upper southeastern town of 20,000 are not representative of family medicine residents nationally. So, our findings should be generalized only to similar sites and residents with similar demographics. Our results are also subject to type 2 error because of small group sizes, although ours is comparable in size to most previous studies. A larger sample size may reveal differences in JSE scores vs. traditional demographics, and a broader data element inclusion might shed light on potential confounders. The fact that a significant association was found with rural upbringing despite the small sample size is remarkable.

Some residents may have provided more socially desirable responses, but this should not have differed in the rural vs. urban groups. Both declined after the PG-1 year, and all responses were anonymized. Although the means were significantly different, the variation in scores was considerable within each subgroup. A strength of our study is that all previous reports were cross-sectional at a single point in time in urban environments. Our study used a longitudinal design with multiple cross-sectional measurements over time in a rural site. Most previous studies had a response rate ranging from $35 \%$ to $80 \%$. A loss rate of only $1 \%$ is a strength of our study.

Perhaps the most critical limitation is whether the JSE measures what experienced physicians recognize as empathy. We are currently studying this issue in our population of residents and faculty. Even more important is whether physicians with higher JSE scores are perceived by their patients as more empathetic. This is an area of very active debate among medical educators, and the majority agree that currently, the JSE is the best measure we have. ${ }^{26-}$ 28

So, what do our findings mean? The hypotheses generated from our data could include that rural students are more resourceful and resilient to have made it to residency despite educational and socioeconomic disadvantages. If this is true, it makes an even stronger case for admitting more rural students into medical school if our goal is to produce empathetic physicians. Our study design does not allow us to make that case. We are currently using other contemporaneous measures to describe the subset of residents who rebound from any loss of measured empathy during the PG-1 year. In our previous report, when our residents in focus groups were asked to rank ten issues that would explain an individual resident's score on the JSE, first by a wide margin was "general outlook on life." ${ }^{15}$ This merits further study.

The other possibility is that rural residents working in a rural environment are more comfortable with the aspects of daily living outside of the working environment, allowing an opening to recover or maintain measures of empathy. An apparent potential explanation is that a physician might find it easier to empathize with patients who share their speech patterns and sociocultural background. If this is true, we need more rural residencies and more pathway programs to get rural students into medical school to address rural physician shortages. Our study design does not allow us to make that case either, and we continue focus groups among our residents to discern possible explanations. We also invite others to study empathy in their residencies and potentially pool data, allowing a broader and larger population to be reported.

\section{CONCLUSION}

In this preliminary study in our group of family medicine residents in our rural site, we found that those with rural upbringing had similar JSE scores as they began residency and showed a similar decline after the PG-1 year compared to their urban classmates. Then, after the PG-2 and PG-3 years, those with rural upbringing showed a significant rebound of JSE scores equivalent to those at baseline, while those with urban upbringing did not recover and declined slightly. 


\section{AUTHOR AFFILIATIONS}

1. University of Louisville School of Medicine Trover Campus at Baptist Health Deaconess Madisonville

2. University of Louisville School of Medicine, Office of Graduate/Undergraduate Medical Education

\section{REFERENCES}

1. Crandall LA, Dwyer JW, Duncan RP. Recruitment and retention of rural physicians: issues for the 1990s. J Rural Health. 1990 6(1):19-38. doi:10.1111/j.1748-0361.1990.tb00188.x.

2. Brooks RG, Walsh M, Mardon RE, Lewis $M$, Clawson $A$. The roles of nature and nurture in the recruitment and retention of primary care physicians in rural areas: a review of the literature. Acad Med. 2002 77(8):790-8. doi:10.1097/00001888-200208000-00008.

3. Orzanco MG, Lovato C, Bates J, Slade S, Grand'Maison P, Vanasse A. Nature and nurture in the family physician's choice of practice location. Rural Remote Health. 2011 11(3):1849.

4. Crump WJ, Fricker RS, Ziegler CH, Wiegman DL. Increasing the Rural Physician Workforce: A Potential Role for Small Rural Medical School Campuses. J Rural Health. 2016 32(3):254-259. doi:10.1111/jrh.12156.

5. Smucny J, Beatty P, Grant W, Dennison T, Wolff LT. An evaluation of the Rural Medical Education Program of the State University Of New York Upstate Medical University, 1990-2003. Acad Med. 2005 80(8):733-8. doi:10.1097/00001888200508000-00006.

6. Zink T, Power DV, Finstad D, Brooks KD. Is there equivalency between students in a longitudinal, rural clerkship and a traditional urban-based program? Fam Med. 2010 42(10):702-6.

7. Crump WJ, Fricker RS, Ziegler C, Wiegman DL, Rowland ML. Rural track training based at a small regional campus: equivalency of training, residency choice, and practice location of graduates. Acad Med. 2013 88(8):1122-1128. doi:10.1097/ACM.0b013e31829a3df0.

8. Rabinowitz HK, Diamond JJ, Markham FW, Wortman JR. Medical school programs to increase the rural physician supply: a systematic review and projected impact of widespread replication. Acad Med. 2008 83(3):235-43. doi:10.1097/ACM.0b013e318163789b.

9. Frisch L, Kellerman R, Ast T. A cohort study of family practice residency graduates in a predominantly rural state: initial practice site selection and trajectories of practice movement. J Rural Health. 2003 19(1):47-54. doi:10.1111/j.1748-0361.2003.tb00541.x.

10. Maudlin RK, Newkirk GR. Family Medicine Spokane Rural Training Track: 24 years of ruralbased graduate medical education. Fam Med. 2010 42(10):723-8.

11. Ross R. Fifteen-year outcomes of a rural residency: aligning policy with national needs. Fam Med. 2013 45(2):122-7.

12. Hojat $M$, Vergare MJ, Maxwell $K$, et al. The devil is in the third year: a longitudinal study of erosion of empathy in medical school. Acad Med. 2009 84(9):1 182-91. doi:10.1097/ ACM.0b013e3181b17e55.

13. Hojat M, Gonnella JS. Eleven Years of Data on the Jefferson Scale of Empathy-Medical Student Version (JSE-S): Proxy Norm Data. and Tentative Cutoff Scores. Med Princ Pract. 2015 24(4):344-50. doi:10.1159/000381954

14. Crump WJ, Ziegler CH, Fricker RS. A Longitudinal Measure of Medical Student Empathy at a Regional Campus: Are we different? Could this be a valuable evaluation method for curriculum change? J Regional Medical Campuses. 2021 4(1). doi:10.24926/jrmc.v4i1.3475.

15. Crump W, Ziegler C, Fricker R. Does empathy really decline during residency training? $\mathrm{A}$ longitudinal look at changes in measured empathy in a community program. J Regional Medical Campuses 2021. 4(4). doi: 10.24926/jrmc. v4i4.4206.

16. Crump W, Ziegler C, Fricker R. A Residency Professional Identity Curriculum and a Longitudinal Measure of Empathy. J Regional Medical Campuses. 2018 1(4). doi:10.24926/jrmc. v1i4.1353.

17. Martin D. A Short History of Trover Clinic with Commentary. McClanahan Publishing House; 1989.

18. Mangione S, Kane GC, Caruso JW, Gonnella JS, Nasca TJ, Hojat M. Assessment of empathy in different years of internal medicine training. Med Teach. 2002 24(4):370-3. doi:10.1080/01421590220145725. 
19. Foreback J, Kusz H, Lepisto BL, Pawlaczyk B. Empathy in Internal Medicine Residents at Community-based Hospitals: A Cross-sectional Study. J Med Educ Curric Dev. 2018 5:1-4. doi:10.1177/2382120518771352.

20. Wolfshohl JA, Bradley K, Bell C, et al. Association Between Empathy and Burnout Among Emergency Medicine Physicians. J Clin Med Res. 2019 11(7):532-8. doi:10.14740/jocmr3878

21. Rural-Urban Continuum Codes [Internet]. Washington, DC: USDA ERS. 2020. [Cited 2021 Aug 6]. Available from: https://www.ers.usda.gov/ data-products/rural-urban-continuum-codes.

22. Wickham H, Chang W., Henry L, Pendersen TL, Takahasi K, Wilke, C. Woo, K. Yutani H., Dunnington, Y. Elegant Graphics for Data Analysis Ggplot Website [Internet]. Tidyverse.org. [cited 2021 Feb 3]. Available from: https://ggplot2. tidyverse.org.

23. WWAMI Rural Health Research Center. Policy Brief. Family Medicine Rural Training Track Residencies: 2008-2015 Graduate Outcomes [Internet]. 2016 Feb. [cited 2021 Oct 21]. Available at https://depts.washington.edu/fammed/rhrc/ wp-content/uploads/sites/4/2016/02/RTT Grad Outcomes PB 2016.pdf.

24. Colliver JA, Conlee MJ, Verhulst SJ, Dorsey JK. Reports of the decline of empathy during medical education are greatly exaggerated: a reexamination of the research. Acad Med. 2010 85(4):588-93. doi:10.1097/ ACM.0b013e3181d281dc.

25. Ferreira-Valente A, Monteiro JS, Barbosa RM, Salgueira A, Costa P, Costa MJ. Clarifying changes in student empathy throughout medical school: a scoping review. Adv Health Sci Educ Theory Pract. 2017 22(5):1293-313. doi:10.1007/s10459-0169704-7.

26. Sinclair S, Kondejewski J, Jaggi P, Dennett $L$, Roze des Ordons AL, Hack TF. What Is the State of Compassion Education? A Systematic Review of Compassion Training in Health Care. Acad Med. 2021 96(7):1057-70. doi:10.1097/ ACM.0000000000004114.

27. Costa-Drolon E, Verneuil L, Manolios E, RevahLevy A, Sibeoni J. Medical Students' Perspectives on Empathy: A Systematic Review and Metasynthesis. Acad Med. 2021 96(1):142-54. doi:10.1097/ACM.0000000000003655.
Bragan L, Calabrese LH. Does Empathy Decline in the Clinical Phase of Medical Education? A Nationwide, Multi-Institutional, Cross-Sectional Study of Students at DO-Granting Medical Schools. Acad Med. 202095

28. Hojat M, Shannon SC, DeSantis J, Speicher MR, 